\section{DNA is all the RAGE}

Activation of the receptor for advanced glycation end products (RAGE) by ligands results in proinflammatory responses. As its name implies, RAGE can bind glycans modified by the Maillard reaction, the nonenzymatic process that leads to browning of food during cooking; such glycated products can also form in the body, particularly in chronic diseases such as diabetes. Other RAGE ligands have been described, including amyloid- $\beta$ fibrils and the nuclear protein HMGB1. Previous work showed that nucleic acids induced inflammatory responses through binding of intracellular receptors in the cytosol or in endosomal compartments. Now Latz, Xiao and colleagues report that RAGE binds extracellular DNA molecules, promoting their uptake into cells. The authors initially observed that cells overexpressing RAGE could accumulate DNA on their surfaces. Purified RAGE could bind different forms of DNA or RNA, with apparent affinities in the micro- to nanomolar range. Two crystal structures of the RAGE extracellular domain in complex with 22-bp DNA sequences were solved, revealing a RAGE homodimer with DNA bound in a positively charged groove formed between the two monomers. In both structures, RAGE formed contacts exclusively with the phosphate backbone of the DNA molecule, thus explaining the lack of sequence specificity. Next, the authors explored the functional consequences of the RAGE-DNA interaction. In addition to binding DNA, cells expressing RAGE were able to take up higher levels of DNA than control cells. Furthermore, both receptor and DNA could be observed in early- and late-endosomal compartments, where the DNA receptor TLR9 is located. RAGE could associate with TLR9, as seen by coimmunoprecipitation; moreover, RAGE increased the level of TLR9 activation by low amounts of DNA. Finally, the authors show that mice lacking RAGE have impaired inflammatory responses in the lungs after intranasal administration of DNA and exhibit reduced influx of neutrophils and lower levels of proinflammatory cytokines and chemokines. Altogether, the work indicates that RAGE delivers extracellular DNA into the endosomal compartment to activate TLR9 and has an important role in promoting inflammatory responses to DNA in vivo. (J. Exp. Med. doi:10.1084/jem.20120201, 30 September 2013)

IC

\section{Segregating meiotic chromosomes}

Translational control is known to be important for regulating the meiotic segregation pattern during gametogenesis, but insights into the precise mechanisms involved have been lacking. B-type cyclin CLB3 is a critical determinant of gametogenesis (known as sporulation in yeast), and its presence is restricted to meiosis II through translational repression during meiosis I. To elucidate the mechanism of translational control, Amon and colleagues found the meiosis-specific Ime2 kinase to be responsible for regulating translational activation of $C L B 3$. Interestingly, mRNA deep sequencing and ribosome profiling of synchronized meiotic budding-yeast cell cultures revealed a gene cluster within which patterns of translation were very similar to those of CLB3. Ime2 also governed translation of this cluster, suggesting a common underlying mechanism. The authors hypothesized that Ime2 inhibits the activity of a translational repressor and identified the meiosis-specific RNAbinding protein Rim4 as an Ime2 substrate. Indeed, Rim4 inhibited translation of CLB3 during meiosis I by interacting with the $5^{\prime}$ UTR of $C L B 3$, and in light of previous work, the authors speculated that Rim4 probably regulates translation at the level of initiation. Although Ime2 kinase activity was low during meiosis I, it rose at the onset of meiosis II and induced a decrease in Rim4 protein levels. It is possible that Ime2-dependent phosphorylation of Rim4 targets the protein for degradation, but Ime2 phosphorylation might also affect the RNAbinding affinity of Rim4. Given its central role in gametogenesis, it will be important to gain further insight into how Ime2 expression and activity are regulated. (Genes Dev. 27, 2147-2163, 2013) AH

\section{Please pass the salt}

Tropisms are directional growth responses that allow plants to adjust to changes in their environment. Their underlying mechanisms involve intracellular redistribution of plant hormones, called auxins, to restrict cell elongation to one side of the shoot or root and thus cause it to bend toward or away from the signaling source in response to gravity, water and light. Now Testerink and colleagues show that plants use a similar mechanism, which they call halotropism, to avoid excess salt. They observed that Arabidopsis and tomato seedlings direct root growth away from $\mathrm{NaCl}$ gradients assembled in agar media or in soil. Auxin-responsive reporter constructs revealed that auxin selectively accumulates within cells of the lateral root tip on the side opposite the high salt; chemical disruption of this redistribution eliminates the halotropic response. The differential accumulation of auxins during plant development is partially mediated by PINFORMED auxin efflux carriers (PINs) in the plasma membrane. Two members of this family, PIN2 and PIN3, control auxin accumulation in the root in response to gravity signaling. Monitoring of the fate of PIN-GFP fusions in seedlings reveals that cell-surface localization of PIN2 but not of other carriers is reduced in root epidermal cells that face elevated salt levels. The authors find that directional root growth is modulated by PIN2 endocytosis, not degradation, as it is in response to gravity signals, results further suggesting that $\mathrm{NaCl}$ triggers a distinct signaling pathway. Moreover, PIN2's subcellular relocalization is not induced by mannitol, indicating that halotropism is distinct from the osmotic-stress response. PIN2 internalization and halotropism are both blocked by chemical inhibition of clathrin assembly. Indeed, differential clathrin recruitment to the plasma membrane is induced by salt treatment and is controlled by the phosphatidic acidgenerating enzyme phospholipase D (PLD). PLD inhibitors or mutations that reduce its activity compromise clathrin recruitment and disrupt PIN2 internalization in response to salt stress. That PLD is also implicated in gravity, water and osmotic-stress responses suggests that its fluctuations may serve to integrate multiple environmental signals to optimize root growth in response to changing conditions. (Curr. Biol. doi:10.1016/j.cub.2013.08.042, 21 October 2013) 